\title{
COMPETITIVE SITUATION IN THE RUSSIAN FEDERATION: MODERN TRENDS AND DEVELOPMENT PROBLEMS
}

\author{
Yakovenko N. V. Doctor of Geographical Sciences, Professor, \\ Komov I. V. PhD in Geography, Associate Professor, \\ Matyukhin V. Master, \\ Zigunova A. Master
}

Russia, Voronezh, Voronezh State University

DOI: https://doi.org/10.31435/rsglobal_ijite/01062018/5668

\section{ARTICLE INFO}

Received 09 April 2018

Accepted 11 May 2018

Published 01 June 2018

\section{KEYWORDS}

competitiveness,

Russia,

region,

globalization,

innovation,

economic crisis

\begin{abstract}
The most important element constituting the basis for the functioning of the modern economy of Russia and ensuring the ongoing development of market relations is the competition, understood in a broad sense as a process of rivalry between economic agents, leading to a change in product handling conditions on the relevant market. 2015 was held in Russia under the influence of a number of factors, which contain signs of an economic crisis, which is a condition that affects the development of key segments of the domestic economy and determines Russian policy. Mutual sanctions from the European Union and Russia have caused some changes not only in the economy of the country as a whole, but also in the economy of its individual regions. The Russian economy was faced with the need for a very short time to make a leap in increasing the competitiveness of its products, as well as import substitution, which would normally take many years. In such conditions, the key role of the state is to support not only large branches of the economy and the defense sector, but also to develop a strategy for developing competitive relations in the sphere of small and medium-sized businesses. The article examines the main problems of ensuring Russia's competitiveness, the reasons for the low efficiency of the national innovation system.
\end{abstract}

Глобализация как мировой процесс активно начала распространяться на рубеже веков, а это в свою очередь требует формирования соответствующего уровня конкурентоспособности любого региона. Социально- экономический смысл термина «конкурентоспособность» тесно связан с понятием «конкуренция». Первым это понятие ввел английский экономист, родоначальник классической школы Адам Смит в середине XVII века, называя ее «невидимой рукой» рынка. Современный экономический словарь дает следующее определение: «Конкуренция - состязание между производителями и продавцами товаров, а в общем случае между любыми экономическими, рыночными субъектами; борьба за рынки сбыта товаров с целью получения более высоких доходов, прибыли и других выгод. Конкуренция представляет цивилизованную, легализованную форму борьбы за существование и один из наиболее действенных механизмов отбора и регулирования в рыночной экономике».

Основу конкурентоспособности национальной экономики составляет формируемая государством среда, в которой конкурируют фирмы. Фирмы, которые и берутся за единицу анализа, входят в состав отраслей, соединяемые в экономику страны в целом. Конкурентоспособность стран базируется на развитии высокотехнологичных отраслей. Их состав и источники конкурентного преимущества отражают уровень развития национальной экономики.

Среда формируется из четырех свойств, которые являются детерминантами разработанного М. Портером «национального ромба» (рис.1).

В формирующемся рыночном пространстве России участвуют все его субъекты. Их интересы тесно переплетены и образуют конкурентную среду, в которой выиграть может только тот субъект, которые имеет наиболее надёжные конкурентные преимущества, обеспечивающие ему благоприятные условия для осуществления предпринимательской и коммерческой деятельности. 
В Отчете глобальной конкурентоспособности 2016-2017 гг., который подготовлен Всемирным экономическим форумом, показатели конкурентоспособности России улучшились: 43-е место из 138 стран.

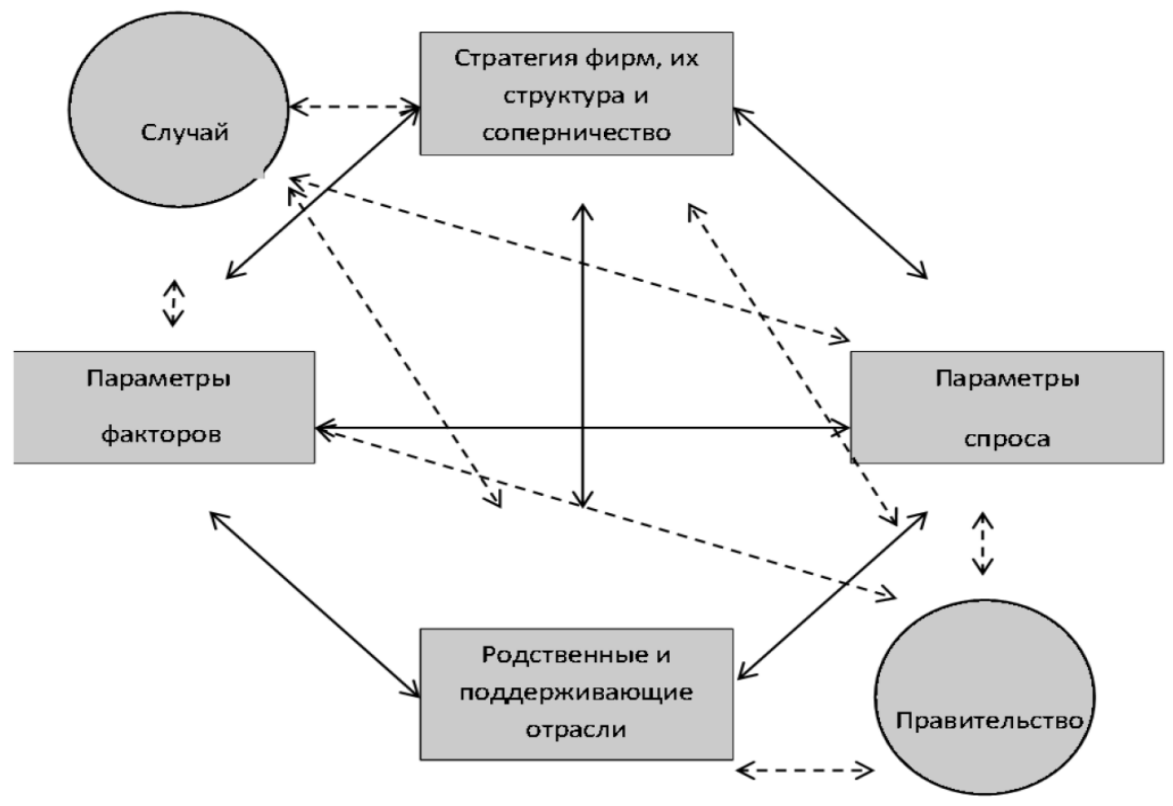

Рис. 1. Детерминанты конкурентного преимущества странь

На первых местах «Глобальном индексе конкурентоспособности» (ГИК), ВЭФ Швейцария, Сингапур и США. Рассматривая основные тенденции социально-экономического развития России, отметим следующее:

1) снижение ВВП на $0,2 \%$,

2) незначительный рост индексов промышленного производства (на $1,1 \%$;

3) незначительный рост продукции сельского хозяйства $(4,8 \%)$;

4) снижение индекса обрабатывающего производства - 0,1\%);

5) инфляция составила 5,4 \% (2015 г. - 12,9\%);

6) потребительские цены выросли на 7,1 \% (2015 г. 5,5 \%);

7) потребительский спрос стал отрицательным и составил - 3,8 \%;

8) общее количество организаций сократилось на $6 \%$.

То есть, исходя из проведенного анализа социально-экономического развития России, можно отметить, что в состоянии конкуренции в Российской Федерации в 2016 г. не произошло каких-либо значимых сдвигов для ее оздоровления и развития.

При чем, многие опасные тенденции в российской экономике, которые создают непреодолимые барьеры в развитии конкуренции не были преодолены государством в 2016 г., а напротив, усугубились (рис. 2).

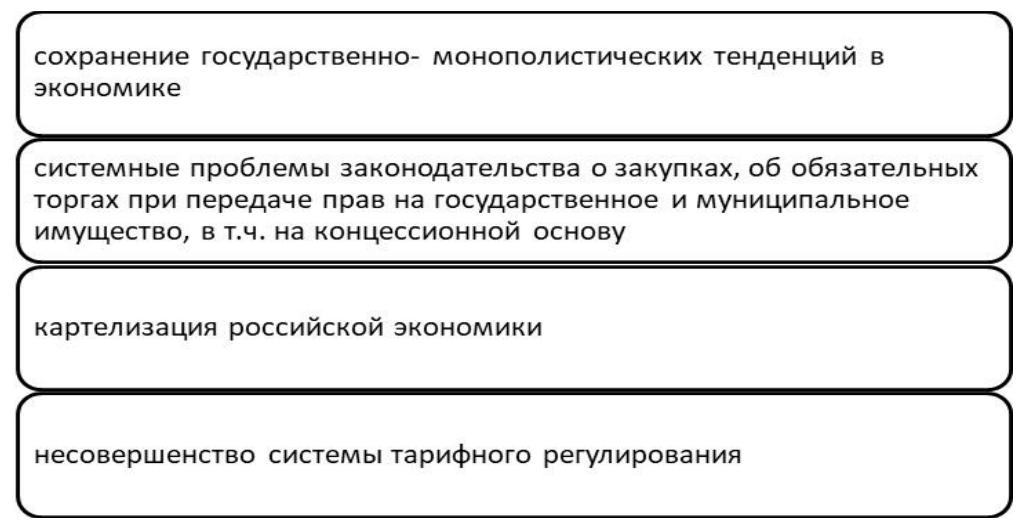

Рис. 2. Ключевые проблемами, препятствующчие развитию экономики и конкуренциии 
Таким образом, российские регионы должны постоянно находить новые инструменты управления и рычаги повышения конкурентоспособности, а также новые источники конкурентных преимуществ, так как в современных реалиях глобализирующегося мира они просто без этого не выживут.

Считаем, что одним из общепризнанных и перспективных направлений в этой ситуации может выступить инновационная деятельность, которая формируется в научно-технической, интеллектуальной и производственной сферах региона.

\section{REFERENCES}

1. Vinokurtseva, E. A. (2017) Konkurentosposobnost regionov Rossii voprosy ekonomiki i upravleniya [Competitiveness of Russian regions in economic and management issues] 2, 88-91. Retrieved 08 May 2018 from: https://moluch.ru/th/5/archive/58/2391/[in Russian].

2. Kiperman, G. Ya. (2012) Sovremennyj ekonomicheskij slovar [Modern Economic Dictionary Moscow: Economy, 255 p. [in Russian].

3. Porter, M. (2016) Meždunarodnaâ konkurenciâ: konkurentnye preimuŝestva stran [International competition: competitive advantages of countries] Moscow: Alpina Publisher, 127 p. [in Russian].

4. Yakovenko, N. V., Komov, I. V., Didenko, O. V., Drobyshev, E. A. (2015) Kontseptual'nye aspekty formirovaniya i razvitiya klasterov v sotsial'no-ehkonomiko-geograficheskoj sisteme regiona [Conceptual aspects of formation and development of clusters in the socio-economicgeographical system of the region], Problems of regional ecology, 6, 61-66. [in Russian].

5. Yakovenko, N. V., Komov, I. V., Didenko, O. V (2017) Strategičeskaâ konkurentosposobnost' regiona: teoretičeskie aspekty issledovaniâ [Strategic competitiveness of the region: theoretical aspects of the study], Science of the Krasnoyarsk6, 1-3, 93-96. [in Russian]. 